


\section{UNGARISCHE JAHREÜCHER}

ZEITSCHRIFT für die kulturellen, sozialen und wirtschaftlichen Fragen Ungarns und seiner Nachbarländer. - Begründet von ROEERT GRAGGER. Unter Mitwirkung von W. BANG, Z. v. GOMBOCZ, E. LEWY, K. SCHUNEMANN, herausgegeben von dem Direktor des Ungarischen Instituts an der Universität Berlin JULYUS v. FARKA S.-Die Jahrbücher erscheinen viermal jährlich. Bd. I, Heft 1-4: RM. 12.-; geb. RM. 13.50 Bd. II, Heft 1-4: RM. 12.-; geb. RM. 13.50 Bd. IU, Heft 1-4: RM. 12.-; geb. RM. 14.- Bd. IV. Heft 1-4: RM. 12.二; geb. RM. 14.Bd. V. Heft 1-4: RM. 16.-; geb. RM. 18.- Bd. VI. Heft 1-4: RM. 20.; geb. RM. 22.Bd. VII, Heft 1-4: RM. 24.-; geb. RM. 26.- Bd. VIII, Heft 1-4: RM. 24.-; geb. RM. 26.Bd. IX, Heft 1-4: RM. 24. - geb. RM. 26.- Bd. X, Heft 1-4: RM. 24.- geb. RM. 28.-

\section{AUS DEM INHALT DER BISHER ERSCHIENENEN BÄNDE: Sprachwissenschatt}

BANG, W.: Türkisches Lehngut im Mandschurischen (IV). - : Turkolog. Briefe aus dem Ungar. Inst. (V, VII, X). BRANDL, A.: Der Name Magyar bei König Alfred (III). BROCKELMANN, C.: Naturlaute im Mitteltürkischen (VIII). GIESE, F.: Zum Wortschatz der altosman, anonymen Chroniken (VIII). GOMBOCZ, Z.: Geschichte der urungar. a-Laute (VIII). - : ther die Haupttypen der ungar. Verbalformen (X). GRAGGER, R.: Zur Geschichte der ugrofinn. Sprachwissenschaft (IV). GYORFFY, St.: Rumänische Ortsnamen (VI). JOKL, N.: Die magyar. Bestandteile des alb. Wortschatzes (VII). JUNKER, H.: Türk. Simnu ,Ahriman“(V). - : Neupers. asan leicht" usw. (V). LEWY, E.: Kurze Betrachtung der ungar. Sprache (IV) - : Arisch-Finno-Ugrisches (VI). - : Possessivisch und passivisch (VIII). - : Wogulische Vorstudien (X). LOSONCZY, Z.: Die ungar. Sprachwissenschaft r910 bis 2 I (II). MARKWART, J.: Np. ädina, ,Freitag' (VII). MEZGER, F.: Altgerm. Zeugnisse zu ost- und nordeurop. Völker- und Landernamen (II). MLADENOV, St.: Zur Erkłårung der sogenannten Buela-Inschrift des Goldschatzes von Nagy Szent-Miklós (VII). MOÓR, E.: Ungarische Flußnamen (VI). NÉMETH, J.: Die petscheneg. Stammesnamen (X). PAIS, D.: Die altungar. Personennamen (III). POPPE, N.: Altaisch und Urtürkisch (VI) - : Die türkischen Lehnwörter im Tschuwassischen (VII). RACHMATULLIN, G. R.: Die Hilfsverben und Verbaladverbien im Altaischen (VIII). SCHAEDER, H. H.: Zur Beschriftung des Schatzfundes von Nagy Szent-Miklós (V). SCHULZE, W.: Zum Tocharischen (VII). - : Osteuropăisches (VIII). SETALA, E.: Ein vorarisches ev. urindogerm. Kulturwort im FinnischUgrischen (VII). - : Einige vor-und urarische-er-und -r-Wörter in den fiugr. Sprachen. (VIII). SZINNYEI, J.: Die Ungar. Akademie der Wissenschaften und die ungar. Sprachwissenschaft (VI). THIENEMANN, T.: Die deutschen Lehnwörter der ungar. Sprache (II). THOMSEN, V.: Aus Ostturkestans Vergangenheit (V). TREML, L.: Die ungar. Lehnwörter im Rumänischen I-II (VIII, IX). WINKLER, H.: Die altaischen Sprachen (IV).

\section{Geschichte und Hilfswissenschaften}

ALFÖLDI, A.: Der Untergang der Römerherrschaft in Panncnien I-III (III, IV). ANGYAL, D.: Das österr. Staats- und Reichsproblem(III). ARRAS, P.: Regestenbeiträge zur Geschichte des Mathias I. Corvinus (IV). BERZEVICZY, A. v.: Der italienische Feldzug von 1859 und Bachs Sturz (VI). BRINKMANN, C.: Bulgarischungarische Beziehungen im 5. bis II. Jh. (II). BRUCCKNER, A.: Zur Geschichte der Slowakei (VI). BUCHNER, M.: Um das Nibelungenlied (IX). FEHÉR, G.: Ungarns Gebietsgrenzen in der Mitte des ro. Jh. (II). GYALÓKAY, E. v.: Die Schlacht bei Mohács (1526) (V1). HOLIK, Fl.: Die erste gelehrte Geselischaft in Ungarn (III). HOLTZMANN, W.: Papst Alexander III. und Ungarn (VI). HOMAN. B.: Der Ursprung der Siebenbürger Székler (II). - : Geschichtliches im Nibelungenlied (III). KAROLYI, A.: Stephan Széchenyis beschlagnahmte Schriften (II) LINTZEL, M.: Theudebert I. und die Sachsen in Pannonien (X). LUKINICH, E. PreuBische Werbung in Ungarn $(\mathrm{x} 722-40)$ (VI). MALYUSZ, E. v.: Die Entstehung des Komitates Túróc (I). MOÓR, E.: Die deutschen Spielleute in Ungarn (I). - : Anschauungen von der Urheimat der Ungarn, im Mittelalter und bei den Humanisten (VII). MORAVCSIK, J.: Zur Geschichte der Onoguren (X). MORDTMANN, J. H.: Osmanische Historiographie und Urkundenlehre (VIII). MÖTEFINDT, H.: Der Schatzfund von Nagy-Szent-Miklós (V). OREND, M., Die ältesten von Stammes-, Gebiets- und Ortsnamen abgeleiteten Familiennamen der Siebenbürger Sachsen (IX). RELKOVIC, N.: Aus dem Leben der sieben "niederungar. Bergstådte" im r4. bis 17. Jh. (VI). SCHMIDT, L.: Die Ostgoten in Pannonien (VI). - : Franken und Sachsen im 6. Jh. in Pannonien? (IX). SCHUNEMANN, K.: Ungar. Hilfsvölker in der Literatur des deutschen Mittelalters (IV). - : Hunnen und Ungarn (V). -: Die "Römer" des anonymen Notars (VI). TAKÁTS, A.: Ungar. und türk. Berufsschreiber im 16. u. 17. Jh. (I). THIM, J. R.: Die Gründungsversuche Jugoslawiens $1848 / 59$ (I). 


\section{UNGARISCHE BIBLIOTHEK}

Für das Ungarische Institut an der Universität Berlin heraus gegeben von JULIUS VON FARKAS

Erste Reihe

16.

Die ungarischen Stileigentümlichkeiten in den musikalischen Werken

Franz Liszts

Von

Zoltán Gárdonyi

Mit 17 Notenbeispielen

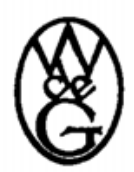

1931

Walter de Gruyter $\mathcal{E}$ Co.

vormals G.J.Göschen'sche Verlagshandlung --J.Guttentag, Verlagsbuch handiung Georg Reimer - Karl J. Trübner - Veit \& Comp.

Berlin und Leipzig 
